\title{
Assessing the Transition From Pre-Clerkship to Clerkship in a Four-Year Medical Program: Recommendations for Future Success
}

Neel Mistry ( $\nabla$ nmist066@uottawa.ca )

University of Ottawa

Stefan de Laplante

University of Ottawa

Craig Murray Campbell

University of Ottawa

\section{Research Article}

Keywords: clerkship, clerkship preparedness, transition, curricular reform, medical education

Posted Date: August 5th, 2021

DOl: https://doi.org/10.21203/rs.3.rs-737559/v1

License: (c) (i) This work is licensed under a Creative Commons Attribution 4.0 International License.

Read Full License 


\section{Abstract}

Background: The transition from pre-clerkship to clinical clerkship is a pivotal moment for medical students. At the University of Ottawa Faculty of Medicine, Unit IV and the Link Block are designed to facilitate this transition. Improvements to the current curriculum, specifically in Unit IV, may better prepare students for clerkship. We aimed to summarize existing literary evidence on the transition to clerkship and collect student feedback to generate recommendations for success with regard to clerkship preparedness at the University of Ottawa Faculty of Medicine.

Methods: We conducted a literature search using PubMed, MEDLINE, ERIC, and CINAHL for studies evaluating the transition to clerkship in a four-year medical program. Using this data, we created two different versions of our survey (pre-transition and post-transition) for dissemination to second, third, and fourth-year medical students, plus MD/PhD students, in the Anglophone and Francophone stream. The survey was open for three weeks from October 10 to October 31,2020, with weekly reminders to all eligible participants. Microsoft Excel 2016 was used for data analysis.

Results: We obtained 176 respondents, of which 158 (70\% Anglophone and 30\% Francophone) were included in the analysis. The majority of students were in the MD2023 cohort (40\%) and had completed a 4-year Bachelor's degree (61\%) prior to medical school. Students in the post-transition group were less anxious about the transition to clerkship than their junior colleagues, although differences between streams were marginal. The most notable difference concerning Entrustable Professional Activities was in terms of obtaining a complete history and performing a physical examination, with the post-transition cohort reporting increased competency compared to the pre-transition cohort in the Anglophone stream (2.9/5.0 pre-transition vs. 3.3/5.0 post-transition, +0.33 difference, $p<0.05)$. Top two stressors for incoming clerks were a lack of clinical skills or experience, and lack of clarity around clerkship roles, responsibilities, and expectations.

Conclusion: There is limited training to facilitate a seamless transition for incoming clerks at the University of Ottawa Faculty of Medicine. Changes can be made at the pre-clerkship level in the form of small-group orientation sessions, formative OSCEs, accelerated review of pre-clerkship material, and clerkship simulation sessions.

\section{Background}

Medical students face innumerable challenges as they traverse the medical education continuum [1]. From managing a rigorous academic workload to striking a work-life balance, medical school unfolds an array of firsts. The story is no different after graduating, as resident doctors learn to respond to the demands of post-graduate medical training [2]. The transition from a classroom setting, in pre-clerkship, to a patient-centered clinical learning environment during clerkship, is the first of many such transitions that trainees encounter on their journey to becoming independently licensed physicians [3]. 
In a four-year medical program, offered at all but two Canadian medical schools [4], pre-clerkship comprises the first two years of medical school while clinical clerkship entails the last two years. Traditionally, pre-clerkship uses formal large- (lectures) and small- (workshops and case-based learning) group educational activities to teach clinical and basic science topics, clinical skills, and core physician competencies (communication, professionalism, and ethics), among others. On the other hand, clerkship focuses on the development of practical clinical skills via mandatory and elective placements in hospital and community settings. At the University of Ottawa, the first year of clerkship comprises a series of mandatory rotations. The final year of clerkship comprises some mandatory components but allows greater flexibility for students to organize elective placements. It is in clerkship that medical trainees learn to work under the supervision of a staff physician to provide direct patient care for the first time.

At the University of Ottawa Faculty of Medicine, Unit IV and the Link Block are designed to facilitate the transition from pre-clerkship to clerkship. Unit IV represents the final unit of pre-clerkship stage of the curriculum that is focused on practical symptom-focused clinical learning activities in geriatrics, pharmacology, pediatrics, palliative care, pain management, dermatology, otolaryngology, global health, complementary and alternative medicine, occupational and environmental health, and health systems. In contrast, the Link Block is a three-week formal introduction to clerkship stage of the MD Program. Here, students are introduced to key skills such as explaining their role as a member of an interprofessional healthcare team; infection prevention and control practices; surgical scrubbing; use of personal protective equipment; documenting a clinical encounter in a medical record; ordering laboratory tests and prescription writing; navigating difficult conversations (end-of-life discussion with patients and their caregivers); and performing clinical procedures (IV access, phlebotomy and arterial blood gas performance) [5].

Existing literature suggests that among students who entered clerkship, social dynamics of group work and a lack of clarity around roles and responsibilities seemed to be the biggest concerns $[2,6]$. These findings were confirmed by Wenrich et al., who garnered student and faculty opinion on clerkship preparedness through a self-reported questionnaire [7]. It was found that clerks reported a heightened perception of what was expected of them compared to faculty members. Interventions must be placed to align student and faculty expectations so that incoming clerks do not feel overwhelmed [8-11]. Better communication about clerkship expectations amongst students and faculty members may help to alleviate the obstacles associated with the transition from pre-clerkship to clinical clerkship. An important starting point is to identify how students perceive said transition and what changes can be made to facilitate them in this process.

However, there is limited evidence on the elements that contribute to a successful transition to a clinical learning environment and relevant best practices to achieve such a transition [12-15]. We conducted this study to set a baseline understanding of research done in this field and to identify avenues for future development. In particular, we aimed to summarize existing literature on the transition to clerkship in a four-year medical program and design a survey to obtain student feedback on the effectiveness of our 
approach to preparing students for their transition to clerkship stage of the MD Program at the University of Ottawa Faculty of Medicine.

\section{Methods}

\section{Literature review}

We conducted a literature review to answer the following research questions: Among students in a fouryear medical program (1) What perceptions exist surrounding the transition from pre-clerkship to clerkship, and (2) What interventions exist to improve this transition? From July 1 to August 31, 2020, we searched MEDLINE, MEDLINE In-Process, Embase, PubMed, ERIC, and CINAHL for studies on the transition to clerkship. A search strategy was generated using group consensus to guide the research questions (Figure 1). Following an initial search, we decided to use PubMed as the database of choice as it included all overlapping articles and more. Included articles were written in the English language, published within the last decade (2010-2019), contained an abstract and full text, and involved medical students or faculty members.

Neel Mistry (NM) and Stefan de Laplante (SDL) independently screened articles based on title and abstract to determine eligibility for full-text review. Any discrepancies were resolved at this stage. Articles were screened further according to the criteria in Figure 2. Our search strategy was kept broad to include 231 articles, of which only 7 were included in the literature review.

Data extraction was conducted by NM and cross-referenced by SDL (Appendix A). Any discrepancies were further discussed at this stage. If consensus could not be reached, a third opinion was sought from Craig Murray Campbell (CMC). NM and SDL independently analyzed the data to answer the research questions for this study. Findings were discussed with $\mathrm{CMC}$ through an iterative process to ensure that the study design and analysis met the aims of this study. Our team comprised a medical student (NM) and two physicians (SDL and CMC) with a strong background in medical education.

\section{Survey design}

Our anonymous survey was inspired by findings from Artino et al. [16]. It was divided into four components: student demographics, clerkship preparedness, core competencies, and curricular reform. Under demographics, students were asked to identify their year and stream (Anglophone or Francophone). Students' educational background prior to medical school was also assessed to determine any existing association with the student's perceived transition to clerkship [17]. Under core competencies, we compared students' self-reported competency to perform relevant national Entrustable Professional Activities (EPAs) before and after starting clerkship (Figure 3). We prepared two different versions of the survey: pre-transition (for MD2023 and MD/PhD students who were yet to enter clerkship; Appendix C) and post-transition (for MD2021, MD2022, and MD/PhD students who had entered clerkship; Appendix D). The pre-transition survey assessed students' concerns with starting clerkship, while the post-transition survey focused on changes that could better facilitate this transition. 
Our survey was designed using SurveyMonkey and included multiple-choice, Likert-type, and short-answer questions. Prior to dissemination, both versions of the survey (pre-transition and post-transition) were translated into French for the MD2021, MD2022, MD2023, and MD/PhD students in the Francophone stream. The survey was open from October 10 to October 31, 2020 (3 weeks). Weekly reminders were sent to all students via a faculty listserv.

\section{Statistical analysis}

Anonymous data was imported from SurveyMonkey and analyzed by NM. Microsoft Excel 2016 (Microsoft, Albuquerque, USA) was used to calculate the mean and standard deviation for all Likert-type scale questions. In addition, we ran two-sample t-tests assuming unequal variance to generate $p$-values and determined statistical significance $(p<0.05)$. Comparisons were made between stream (Anglophone vs. Francophone) and cohort (pre-transition vs. post-transition). Qualitative data was obtained by classifying student responses to open-ended questions. NM read and coded individual comments into thematic clusters, while SDL reviewed and affirmed this. A third opinion from CMC was sought in case of disagreement. Comments were classified into one of five categories (roles and responsibilities, curricular changes, clinical skills, administrative, preparation and training), which were generated based on prevalent themes that arose from qualitative analysis. Subgroups for each category were created to better classify student responses.

\section{Results}

Invitations were sent to all first (MD2023, $n=162)$, second (MD2022, $n=163$ ) and third (MD2021, $n=163$ ) year medical students, as well as MD/PhD students $(n=12)$, from both streams at the University of Ottawa. We obtained 176 respondents (37\% response rate; 127 Anglophone and 49 Francophone), of which 6 did not provide consent to participate. Of those who provided consent, 12 respondents (11 Anglophone and 1 Francophone) did not initiate the survey.

\section{Demographics}

Of the 158 respondents included in the study: $n=110(70 \%)$ were in the Anglophone stream and $n=48$ (30\%) were in the Francophone stream. The proportion of respondents from each stream was similar (110 of $331,33 \%$ Anglophone vs. 48 of $149,32 \%$ Francophone). The majority of respondents were in the MD2023 cohort $(n=63,40 \%)$, followed by MD2022 ( $n=56,35 \%)$, MD2021 $(n=33,21 \%)$, and MD/PhD $(n=6$, $4 \%)$. Prior to medical school, $61 \%(n=97)$ completed a 4-year Bachelor's degree and $22 \%(n=35)$ completed a 3-year Bachelor's degree (Appendix E). There was no association between education prior to medical school and perceptions related to the transition to clerkship.

\section{Clerkship preparedness}

Under clerkship preparedness, pre-clerkship students were asked to comment on how they felt with regard to the upcoming transition to clerkship (pre-transition survey), using four Likert-type scale questions ( $1=$ 
strongly disagree and 5 = strongly agree). Students in the Anglophone stream reported greater concerns compared to their Francophone colleagues on all but one question. The difference between both streams ranged from adjusting to the hospital setting ( $+0.3 ; 4.4 \pm 0.8$ Anglophone vs. $4.1 \pm 0.9$ Francophone) to being effectively prepared for clerkship (-0.2; $4.1 \pm 0.9$ Anglophone vs. $4.3 \pm 0.6$ Francophone). None of these findings achieved statistical significance.

MD2021, MD2022, and MD/PhD students in clerkship (post-transition survey) were asked to reflect on this transition by responding to same four Likert-type scale questions as above ( $1=$ strongly disagree and 5 = strongly agree). Compared to pre-clerkship students, clerks reported feeling less anxious in retrospect. The greatest difference between streams was with regard to adjusting to the hospital setting $(+0.3 ; 3.5 \pm$ 1.0 Anglophone vs. $3.2 \pm 1.1$ Francophone) and the least difference was for clarity of clerkship roles and responsibilities $(-0.1 ; 3.6 \pm 1.1$ Anglophone vs. $3.7 \pm 1.2$ Francophone). None of these findings achieved statistical significance.

Compared to Anglophone students in the pre-transition cohort, those who had transitioned to clerkship (i.e., post-transition cohort) reported increased competency on most EPAs. This was especially true with regard to taking a complete patient history and performing a physical examination tailored to the patient's clinical situation $(+0.33 ; 3.26$ post-transition vs. 2.93 pre-transition, $p<0.05)$. Students in the pretransition group felt equally or more confident than those in the post-transition group with regard to interpreting and explaining common diagnostic tests $(-0.02 ; 2.98$ post-transition vs. 3.00 pre-transition, $p=0.92)$, designing a management plan for patients $(-0.08 ; 2.59$ post-transition vs. 2.67 pre-transition, $p=0.66)$, and breaking bad news $(-0.07 ; 2.70$ post-transition vs. 2.77 pre-transition, $p=0.75)$. These results were not statistically significant.

In the Francophone stream, the majority of MD2023 and MD/PhD students who completed the pretransition survey reported feeling equally or more competent than those in the post-transition group. The greatest difference was seen with regard to history taking and patient examination $(-0.22 ; 2.84$ posttransition vs. 3.06 pre-transition, $p=0.26)$, although this was not statistically significant. There was marginal difference with respect to students' ability to order workup based on a differential diagnosis $(-0.20 ; 2.36$ post-transition vs. 2.36 pre-transition, $p=0.40)$. Students in both groups felt equally competent in coming up with a differential diagnosis $(+0.03 ; 2.72$ post-transition vs. 2.69 pre-transition, $p=0.87)$, interpreting and communicating results of a diagnostic test $(+0.09 ; 2.84$ post-transition vs. 2.75 pretransition; $\mathrm{p}=0.71)$, recognizing urgent care and providing management $(-0.05 ; 2.76$ post-transition vs. 2.81 pre-transition, $p=0.87)$, and educating patients on disease management $(-0.04 ; 3.08$ post-transition vs. 3.12 pre-transition, $p=0.86$ ).

Students in the pre-transition and post-transition survey were asked to comment on aspects of the transition to clerkship that they were most concerned about (or are concerned about, in the case of MD2023 and MD/PhD students). Comments were summarized into individual themes and the top two themes for each question are displayed above (Table 3). See Appendix F for a complete list. 
Among pre-clerkship students $(n=56)$, being able to independently assess patients and perform a physical examination was a major concern with regard to the transition to clerkship. A large proportion of them were apprehensive about lacking the skills needed to succeed as clerks $(28 \%)$, given cancellation of clinical placements due to the COVID-19 pandemic. Additionally, incoming clerks felt unsure about their roles and responsibilities (22\%). Students in the MD2021, MD2022, and MD/PhD cohorts who completed the post-transition survey had similar fears when starting clerkship. Anglophone and Francophone students noted that they were unsure about their roles as clerks, and whether or not they were being helpful to their team (34\%). In addition, they stated that a lack of clarity existed with regard to what was expected of them during each rotation. Close to $25 \%$ of clerks were overwhelmed by the amount of content they had to know, including specific physical examinations.

In terms of changes that could better prepare incoming students for said transition, a focus on practical skills and strategies (28\%) along with curricular changes (23\%) were identified to be key. Pre-clerkship students expressed struggling with "soft skills" such as donning and doffing medical equipment, writing discharge notes, filing admission orders, and using EPIC [Electronic Medical Record], but also expressed a desire to learn these. Clerks who were asked to comment on improvements that can be made at the preclerkship level suggested increasing the duration of clinical skills training (23\%) and reinforcing clerkship logistics (17\%). In particular, they felt that more practice with physical exams and case management may be beneficial in simulating the requirements of clerkship. Third and fourth-year medical students suggested that practical skills be prioritized over clinical medicine topics, where possible, so that incoming clerks are able to better excel in their roles. This includes providing training on how to summarize a patient consult, what to check on morning rounds, how to effectively take notes on the wards, and who to contact for various administrative needs.

\section{Discussion}

We conducted a literature review to answer the following research questions: Among students in a fouryear medical program (1) What perceptions exist surrounding the transition from pre-clerkship to clerkship, and (2) What interventions exist to improve this transition? We found that although existing studies have suggested improvements with regard to the transition to clerkship, ours appears to be the first to obtain direct student feedback on this major transition with practical strategies that could enhance this transition period.

In this study, a lack of clinical skills training, limited access to patient-related experiences, limited opportunities to practice clinical skills and the lack of clarity on the roles, responsibilities, and expectations of clinical clerks appeared to be the greatest challenges to a seamless transition to clerkship in the Faculty of Medicine at the University of Ottawa. Students in both language streams felt equally apprehensive about clerkship preparedness. Our study found no association between educational background before medical school and perceptions of the transition to clerkship. We believe this is because the transition to clerkship poses many unique challenges that is independent of students' prior educational background or clinical knowledge. Those who had already entered clerkship reported feeling 
less anxious, in retrospect, than those who were yet to enter clerkship. This insinuates that perceptions and realities were distinct with regard to the transition to clerkship.

Several studies have suggested strategies to ameliorate the transition from pre-clerkship to clerkship. Similar to our findings, Hoffman and Cohen-Osher introduced a new tool in clerkship, called the OneMinute Learner (OML), to encourage discussion of goals and expectations between medical students and faculty members [8]. After implementation, students' awareness of their roles and responsibilities in clerkship increased significantly [8]. Furthermore, Near-Peer Teaching, a strategy where senior students teach junior ones, has been shown to be effective in the transition-to-clerkship by allowing students to build a focused study plan and seek advice from upper-year mentors [9]. At the University of California San Francisco, the implementation of an elective increased competency in history-taking and physical examination among elective students compared to non-elective students [10]. This was supported by research at Brown University where the introduction of a three-week clinical skills program enhanced the transition-to-clerkship among incoming third-year medical students [11].

Recommended changes to the pre-clerkship curriculum to facilitate the transition to clerkship for thirdyear students at the University of Ottawa include providing a brief overview of clerkship roles and responsibilities in the form of case-based learning and small-group sessions [6-8], organizing formative Objective Structured Clinical Examinations (OSCEs) prior to clerkship so that students can become comfortable with independently seeing patients, hosting a faculty-led accelerated review of high-yield preclerkship content $[7,9]$, and providing early exposure to patients in a broad array of clinical settings including in-hospital calls and simulation shifts $[10,11]$. Although a large number of student responses were similar to what is found in the literature, the introduction of formative OSCEs to ameliorate trainees' clinical skills was unique. Moreover, while simulation sessions have already been incorporated at medical schools in the U.S. $[8,9]$, the inclusion of in-hospital calls during pre-clerkship was a suggestion unique to our study.

Our study is not without limitations. With regard to the literature review, our search strategy was to only allow articles published in the last decade. Perhaps including articles prior to 2010 would have provided greater insight into the challenges associated with transition to clerkship and helped inform our survey design. Second, in terms of our survey design, we did not follow all of the steps outlined by Artino et al. to design an effective survey questionnaire [16]. We did not have the financial resources to obtain expert opinion on our survey and had only ten weeks to create the survey, given the time limit of the summer studentship and NM's start of second year of medical school. Most importantly, our study did not monitor the same cohort over time (i.e., throughout their pre-clerkship and clerkship years). As a result, we created two different versions of our survey (pre-transition vs. post-transition) with different target audience for each (MD2023 and MD/PhD for the former vs. MD2021, MD2022, and MD/PhD for the latter) which did not provide an accurate representation of how the same group of students felt before and after undergoing the transition to clerkship.

\section{Conclusion}


The transition from pre-clerkship to clerkship is a pivotal moment for many reasons. Among other challenges, adjusting to the hospital setting and a lack of clarity around clerkship roles and responsibilities can be daunting. Improvements should be made at the pre-clerkship level to include small-group sessions that highlight roles, responsibilities and expectations of clerks, providing more opportunities to practice clinical skills with more formative OSCEs to receive feedback prior to clerkship. Explicit education to focus on the competencies required to complete a history and physical examination, generate a differential diagnosis, develop investigation and management plans and enhance student familiarity with the clinical learning environment including allowing for simulation call shifts are offered as potential options. By providing early and frequent exposure to patients across multiple settings, we hope that students can better navigate the transition to clinical clerkship at the University of Ottawa and other Canadian medical schools.

\section{Abbreviations}

OSCE Objective Structured Clinical Examination

ÉCOS Examen Clinique Objectif Structuré

EPAs $\quad$ Entrustable Professional Activities

AMUHM Association Médicale Universitaire de L'Hôpital Montfort

REB Research Ethics Board

OML One-Minute Learner

NM Neel Mistry

SDL Stefan de Laplante

CMC Craig Murray Campbell

\section{Declarations}

Ethics approval and consent to participate: The study was approved by the Research Ethics Board (REB) at L'Hôpital Montfort (No. 20-21-08-024) and the University of Ottawa Health Sciences Network (No. H-0920-6125). All participants were aware that they were taking part in this research and gave informed consent in addition to confirming that they would allow us to use their collected data anonymously for publication (Appendix B). All data was anonymously collected and analyzed.

Consent for publication: Not applicable.

Availability of data and materials: Appendix A-F. 
Methods: All methods were performed in accordance with the relevant guidelines and regulations.

Competing interests: The authors declare that they have no competing interests.

Funding: NM was supported by a bursary through the University of Ottawa Faculty of Medicine Summer 2020 Studentship. SDL obtained funding support from AMUHM.

Contributions: NM and SDL conceptualized the study and were involved in its design. NM was responsible for data collection and analysis. NM produced the first draft of the paper; both NM and SDL contributed to iterative drafting and initial revisions. All authors (NM, SDL, and CMC) contributed to refinement of the final version of the manuscript and approve of its submission.

Acknowledgements: University of Ottawa Faculty of Medicine translation services for translating both surveys (Appendix B-D) from English to French. Dr. Timothy Wood, Director of Evaluation, University of Ottawa Faculty of Medicine, for guiding the statistical analysis.

Authors' information: NM is an incoming third-year medical student at the University of Ottawa. SDL is the Unit IV (Integration) Leader for the Francophone and Anglophone stream and Lead Physician at UMF Montfort FHO. CMC is the Curriculum Director for Undergraduate Medical Education at the University of Ottawa Faculty of Medicine.

\section{Approval and funding}

Research Ethics Board (REB) approval for this study was obtained from L'Hôpital Montfort (primary review) and the University of Ottawa Health Sciences Network (expedited review).This study was funded by the University of Ottawa Faculty of Medicine Summer 2020 Studentship and the Association Médicale Universitaire de l'Hôpital Montfort (AMUHM).

\section{References}

1. Hill MR, Goicochea S, Merlo LJ. In their own words: stressors facing medical students in the millennial generation. Med Educ Online 2018;23:1.

2. Atherley A, Dolmans D, Hu W, et al. Beyond the struggles: a scoping review on the transition to undergraduate clinical training. Med Educ 2019;53:559-570.

3. Greenberg L, Blatt B. Perspective: Successfully Negotiating the Clerkship Years of Medical School: A Guide for Medical Students, Implications for Residents and Faculty. Acad Med 2010;85:706-709.

4. Raymond JR, Kerschner JE, Hueston WJ, et al. The Merits and Challenges of Three-Year Medical School Curricula: Time for an Evidence-Based Discussion. Acad Med 2015;90:1318-1323.

5. Undergraduate Medical Education (UGME) Committee. uOttawa Faculty of Medicine - MD Program Curriculum Objectives [Internet]. University of Ottawa Faculty of Medicine; 2017 [cited 2020 Jun]. 
Available from:

https://curriculum.med.uottawa.ca/common/documents/Objectives/Clerkship/Link/link_objectives.pdf

6. Surmon L, Bialocerkowski A, Hu W. Perceptions of preparedness for the first medical clerkship: a systematic review and synthesis. BMC Med Educ 2016;16:89.

7. Wenrich M, Jackson MB, Scherpbier AJ, et al. Ready or not? Expectations of faculty and medical students for clinical skills preparation for clerkships. Med Educ Online 2010;15.

8. Hoffman M, Cohen-Osher M. The One Minute Learner: Evaluation of a New Tool to Promote Discussion of Medical Student Goals and Expectations in Clinical Learning Environments. Fam Med 2016;48:222225.

9. Knobloch AC, Ledford CJW, Wilkes S, et al. The Impact of Near-Peer Teaching on Medical Students' Transition to Clerkships. Fam Med 2018;50:58-62.

10. Connor DM, Conlon PJ, O'Brien BC, et al. Improving Clerkship Preparedness: A Hospital Medicine Elective for Pre-Clerkship Students. Med Educ Online 2017;22:1307082.

11. Taylor JS, George PF, MacNamara MMC, et al. A New Clinical Skills Clerkship for Medical Students. Fam Med 2014;46:433-439.

12. O'Brien BC, Poncelet AN. Transition to Clerkship Courses: Preparing Students to Enter the Workplace. Acad Med 2010;85:1862-1869.

13. Turner SR, White J, Poth C, et al. Preparing Students for Clerkship: A Resident Shadowing Program. Acad Med 2012;87:1288-1291.

14. Small RM, Soriano RP, Chietero M, et al. Easing the Transition: Medical Students' Perceptions of Critical Skills Required for the Clerkships. Educ Health (Abingon) 2008;21:192.

15. Cox WJ, Desai GJ. The Crisis of Clinical Education for Physicians in Training. Mo Med 2019;116:389391.

16. Artino AR, La Rochelle JS, Dezee KJ, Gehlback H. Developing questionnaires for educational research: AMEE Guide No. 87. Med Teach 2014;36:463-474.

17. Bosch J, Maaz A, Hitzblech T, et al. Medical students' preparedness for professional activities in early clerkship. BMC Med Educ 2017;17:140.

\section{Tables}

Due to technical limitations, table 1-3 is only available as a download in the Supplemental Files section. 


\section{Figures}

((("clerkship preparedness"[MeSH $\quad$ Major $\quad$ Topic:noexp] $\quad$ OR $\quad$ ("transition"[Title/Abstract] OR "readiness"[Title/Abstract])) AND ("clinical clerkship"[MeSH Terms] OR ("clerkship*"[Title/Abstract] OR "pre clerkship*"[Title/Abstract]))) AND ("curriculum"[MeSH Terms] OR "curricular reform*"[Title/Abstract])) AND ("education, medical, undergraduate"[MeSH Terms] OR ("undergraduate medical education"[Title/Abstract] OR "UGME"[Title/Abstract]))

Figure 1

Search strategy for PubMed.

\begin{tabular}{|c|c|}
\hline $\begin{array}{l}\text { Studies obtained in PubMed } \\
\qquad(\mathrm{n}=237)\end{array}$ & \\
\hline & \multirow{3}{*}{$\begin{array}{l}\qquad \begin{array}{c}\text { Records excluded } \\
(n=162)\end{array} \\
\text { 1. Published prior to 2010 } \\
\text { 2. No abstract or full text } \\
\text { 3. Include other healthcare professionals } \\
\text { 4. Not written in English }\end{array}$} \\
\hline $\begin{array}{l}\text { Retrieved after application of filters } \\
\qquad(\mathrm{n}=75)\end{array}$ & \\
\hline$\downarrow$ & \\
\hline \multirow{2}{*}{$\begin{array}{l}\text { Retrieved after screening title and abstract } \\
\qquad(\mathrm{n}=31) \\
\text { 1. Include the following keywords: clerkship } \\
\text { preparedness, transition, clinical clerkship } \\
\text { 2. Focus on specific clerkship rotations }\end{array}$} & \multirow{2}{*}{$\begin{array}{l}\qquad \begin{array}{c}\text { Records excluded } \\
(n=44)\end{array} \\
\text { 1. Target MS1s and/or MS4s } \\
\text { 2. Explore the transition to residency }\end{array}$} \\
\hline & \\
\hline$\downarrow$ & \multirow{3}{*}{ 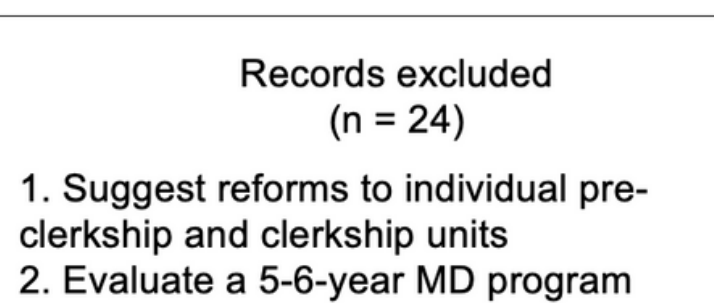 } \\
\hline $\begin{array}{l}\text { Retrieved after screening full text } \\
\qquad(\mathrm{n}=7)\end{array}$ & \\
\hline $\begin{array}{l}\text { 1. Explore the transition to clerkship } \\
\text { 2. Focus on a 4-year medical program }\end{array}$ & \\
\hline
\end{tabular}

Included in literature review

$$
(n=7)
$$

Figure 2

Outline of literature review for studies on the transition to clerkship. 
(1) Obtain a history and perform a physical examination adapted to the patient's clinical situation.

(2) Formulate and justify a prioritized differential diagnosis.

(3) Formulate an initial plan of investigation based on the diagnostic hypotheses.

(4) Interpret and communicate results of common diagnostic and screening tests.

(5) Formulate, communicate and implement management plans.

(7) Provide and receive the handover in transitions of care.

(8) Recognize a patient requiring urgent or emergent care, provide initial management and seek help.

(9) Communicate in difficult situations.

(12) Educate patients on disease management, health promotion and preventative medicine.

Source: Association of Faculties of Medicine of Canada, 2016

\section{Figure 3}

Relevant Entrustable Professional Activities (EPAs).

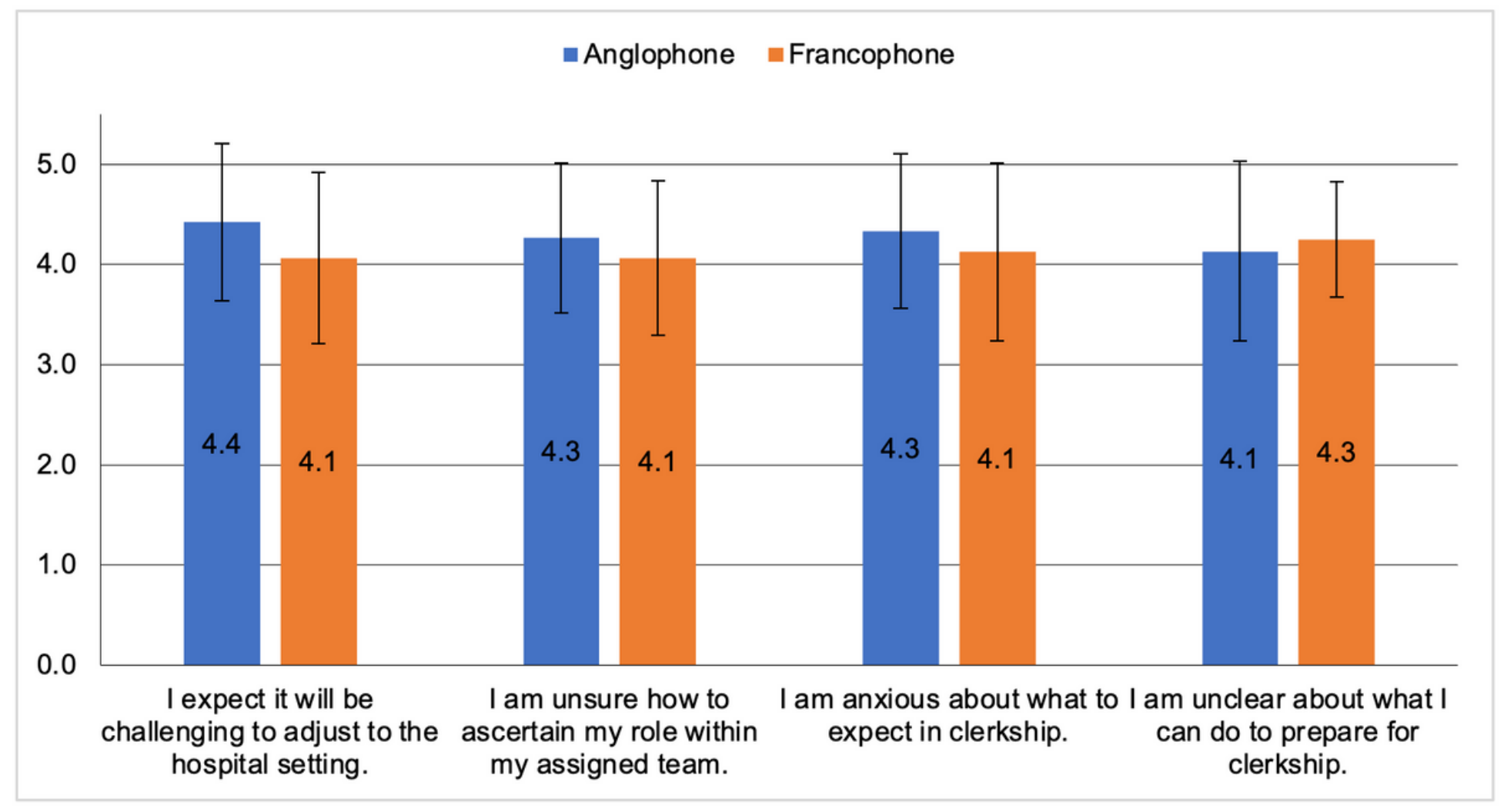

Figure 4

Comparison of student perspectives on the upcoming transition to clerkship between the Anglophone and Francophone stream, with error bars indicating standard deviation. 


\section{- Anglophone $=$ Francophone}

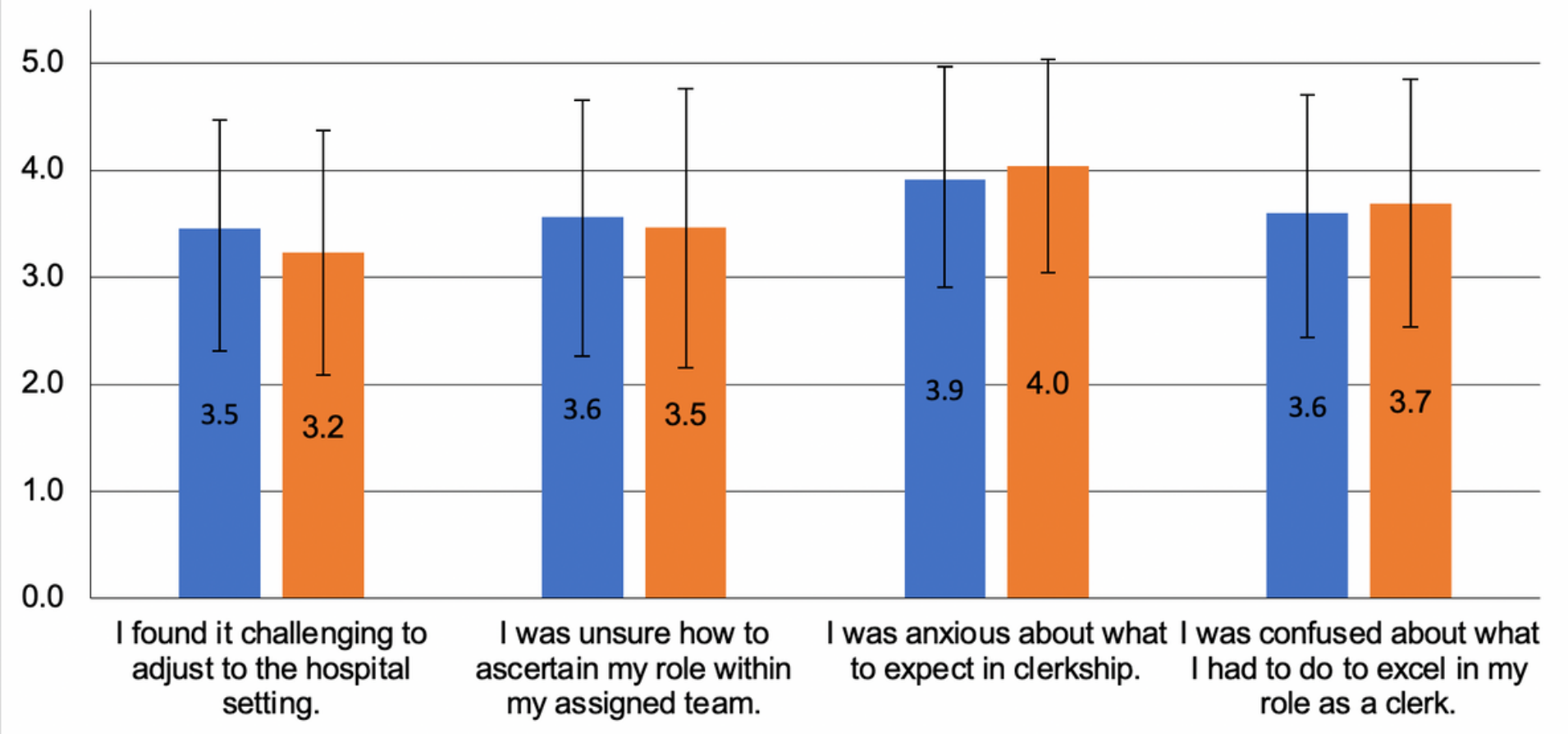

\section{Figure 5}

Comparison of student perspectives on the previous transition to clerkship between the Anglophone and Francophone stream, with error bars indicating standard deviation.

\section{Supplementary Files}

This is a list of supplementary files associated with this preprint. Click to download.

- AppendixAF.docx

- Table1.pdf

- Table2.pdf

- Table3.pdf 